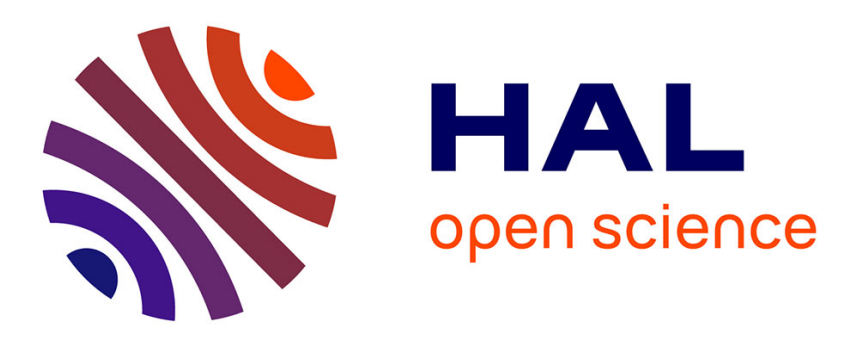

\title{
Choquet Integral based Feature Selection for Early Breast Cancer Diagnosis from MRIs
}

Soumaya Trabelsi Ben Ameur, Florence Cloppet, Sellami Dorra, Laurent

Wendling

\section{- To cite this version:}

Soumaya Trabelsi Ben Ameur, Florence Cloppet, Sellami Dorra, Laurent Wendling. Choquet Integral based Feature Selection for Early Breast Cancer Diagnosis from MRIs. ICPRAM, Feb 2016, Rome, Italy. pp.351 - 358, 10.5220/0005754703510358 . hal-01391970

\section{HAL Id: hal-01391970 \\ https://hal.science/hal-01391970}

Submitted on 4 Nov 2016

HAL is a multi-disciplinary open access archive for the deposit and dissemination of scientific research documents, whether they are published or not. The documents may come from teaching and research institutions in France or abroad, or from public or private research centers.
L'archive ouverte pluridisciplinaire HAL, est destinée au dépôt et à la diffusion de documents scientifiques de niveau recherche, publiés ou non, émanant des établissements d'enseignement et de recherche français ou étrangers, des laboratoires publics ou privés. 


\title{
Choquet integral based feature selection for early breast cancer diagnosis from MRIs
}

\author{
Soumaya Trabelsi Ben Ameur ${ }^{1,2}$, Florence Cloppet ${ }^{1}$, Dorra Sellami Masmoudi ${ }^{2}$ and Laurent \\ Wendling ${ }^{1}$ \\ ${ }^{1}$ Intelligent System of Perception group, LIPADE lab,Paris Descartes University, 45 Saint Pères street, Paris, France. \\ ${ }^{2}$ Computor Imaging and Electronic Systems Group (CIEL), National Engineering School of Sfax (ENIS), Sfax, Tunisia. \\ soumaya.trabelsi_benameur@yahoo.fr, sellamimasmoudidorra@yahoo.com,\{florence.cloppet,lwendlin\}@math-info.univ- \\ paris5.fr
}

Keywords: Breast cancer, computer aided diagnosis, mammography, ultrasound, MRI, dual-energy contrast-enhanced digital mammography, Choquet integral.

\begin{abstract}
This paper focuses on breast cancer of the mammary gland. Both basic segmentation steps and usual features are recalled. Then textural and morphological information are combined to improve the overall performance of breast MRI in a computer-aided system. A model of selection based on Choquet integral is provided. Such model is suitable when handling with a weak amount of data even ambiguous in some extent. Achieved results compared to well-known classification methods show the interest of our approach.
\end{abstract}

\section{INTRODUCTION}

Breast cancer is a malignant tumor of the mammary gland due to the presence of abnormal cells in the breast tissue. In order to reduce the risk of mortality and have an adequate treatment, the doctor can make several different screenings: mammography, ultrasound, breast MRI, Dual-Energy ContrastEnhanced Digital Mammography DECEDM and other additional detection exams. Therefore they are faced with a problem of melting and data decision. Radiologists tend to describe a mass according to its shape, outline, optical density, etc. The diagnosis of breast mass is a difficult task, even for an expert radiologist, because its morphological aspects can be very subtle and hard to diagnose visually. Therefore, Computer-Aided Diagnosis (CAD) has been proposed to support radiologists in the discrimination of malignant and benign lesion. These approaches are usually based on three steps: segmentation of the mass, extraction of characteristics and classification into malignant and benign. Features extracted from breast images are classified into texture features, shape descriptors and kinetic features. There are attributes that are not necessarily relevant for taking the decision, and they can induce an error in the classification thus a multi-criteria selection is necessary. Choquet integral aims at providing a robust decision model even with a small training database (which is the case of our study). This method allows to select and classify the most significant and pertinent attributes.

\section{DETECTION AND ANALYSIS SYSTEM}

Breast pathologies are classified into two types: microcalcifications and breast masses. In our study we are interested to the detection of breast masses. Breast masses are found in several shots. they are generally characterized by their shape and contour. Round or oval masses with well-defined limits are rather benign lesions. While lobulated and undefined limits masses are malignant. Breast density characterizes the heterogeneity of the breast tissue, it has a link with breast cancer because it has an impact on the reliability of the interpretation of medical images. Medical imaging has a crucial role in reducing breast cancer mortality, by making possible early detection through screening. Several breast imaging exists such as mammography, ultrasound, breast MRI and DECEDM, etc. 


\subsection{Mammography}

As the primary imaging modality for early detection and diagnosis of breast cancer, mammography has achieved significant success. A mammography is a breast radiography which allows to obtain images of breast tissue using X-rays to detect anomalies. Radioopaque areas are clear and correspond to the fibroglandular tissue and calcium. A mammogram is performed either for breast cancer screening or to diagnose the presence of symptoms. Mammography is accessible and non-traumatic, it allows a well visualizing of microcalcifications. However, the superposition of different breast tissues limits breast exploration, a high breast density can be the cause of an analytical problem and small tumors could be undetected.

\subsection{Ultrasound}

The ultrasound breast examination is conventionally used in addition to mammography, for a differential diagnosis for cystic lesion/solid lesion and to explore palpable breast lesions without mammography translation. It can be a first-line examination of young women (significant breast density), pregnant women and patients with breast inflammation. Ultrasound is a quick and easy exam even in case of dense breast but exploration of greasy breast is hard. The principal limits of this modality are its non-reproducibility and its sensitivity to motion.

\subsection{Breast MRI}

Breast Magnetic Resonance Imaging is a second line examination, which comes after mammography and often after ultrasound, first for high-risk women because they had many antecedents of cancers in the family, or that they inherited an abnormal gene family. It has also been used to elucidate problems of diagnosis that have not been resolved after the mammogram and ultrasound. The injection of contrast agent might be necessary to visualize areas difficult to explore. The interpretation of breast MRI is based on the perception of the contrast enhancement that varies according to the type of lesion. A quantitative analysis is used to divide the curve, according to the initial peak, into two parts: the initial enhancement and the delayed phase. The initial enhancement can be slow (for benign lesion), medium (intermediate) or fast (suggestive of malignancy). In delayed phase, three types of contrast enhancement are possible: persistent (Benin), tray (intermediate) and washout (malignant). Breast MRI is non-invasive and non-irradiating technique, it allows to study the tumor vasculature independently of breast density. But it is expensive, it has a limited availability, a low specificity and doesn't visualize microcalcifications very well.

\subsection{Dual-energy contrast-enhanced digital mammography DECEDM}

Dual energy contrast enhanced digital mammography DECEDM is a promising new modality that combines mammography and contrast agent injection (iodine). It aims to acquire, in addition to the usual morphological information, data about breast vascularization. DECEDM has a quick and easy image access and analysis and gives a better appreciation of tumor spread in the case of an architectural disorganization the mammography. On the other side, it doesn't detect weak vascularized tumors, an enhancement matrix increases the number of false positives and the fuzzy kinetics increases false negatives.

The complementarity between these different modalities gave us the idea to extract features from more than one modality to have a better recognition and description of the tumor. Because of the nonreproducibility of ultrasound and the fact that information given by mammography could be found on DECEDM (in addition to tumor vascularization), we choose to apply our detection and diagnosis system to two modalities: breast MRI and DECEDM. In this paper we will focus on results obtained from MRIs.

\section{SEGMENTATION AND FEATURE EXTRACTION}

Segmentation is a very important stage in breast cancer diagnostic process. It consists of extracting the pathological area in the image. A good segmentation generates an accurate description of the characteristics of breast pathology. Currently, there are several image processing methods for the segmentation of breast lesions, someone's allow both detection and segmentation. Liney et al. (Liney, 2006) used region growing algorithm for the segmentation of breast MRI. Wang et al. (Wang, 2014) applied region growing algorithm to segment masses in breast MRI detected from a color map. Although region growing was recommended and frequently used for image segmentation, it has a serious limitation. In fact, the growth phase requires 
a criterion of homogeneity. If the seeds are located in a non-homogeneous area, which is the case with malignant lesions, the similarity measure will generate variations which cause the interruption of the growth process. At first, Chen et al. (Chen, 2004) also recommended the use of region growing for segmentation of mammary masses, but then they offered another method of segmentation based on fuzzy C-means clustering (FCM) algorithm and confirmed that the FCM algorithm outperforms the region growing algorithm (Chen, 2006). However, conventional FCM clustering does not integrate information about spatial context which makes it sensitive to noise and imaging artifacts. Conventional smoothing filters can be used to compensate this drawback but they can cause a loss of important image details, especially image boundaries. Some approaches (Zhang, 2003), (Li, 2003) proposed to increase the robustness of FCM to noise but they show considerable computational complexity. Li et al. (Li, 2008) propose an active contour based region model, called the level set method, to segment images with inhomogeneous intensities or objects with hidden or undefined contour. Since its first application for segmentation based contour in early 90s, level set method becomes increasingly popular for image segmentation. Specially, the level set based region allows to partition the image into coherent color, texture and dynamic texture areas. A comparative study between the snake method and the level set method led by Winzenrieth et al. (Winzenrieth, 2003) on hip MRI, proves that the level sets is most appropriate since it is the more reproducible, more accurate and require less manual intervention. To deal with image intensity inhomogeneity, Li et al. (Li, 2011) proposed a novel level set region-based method for image segmentation by deriving a local intensity clustering property from a model of images with intensity inhomogeneities and defining a local clustering criterion function for the intensities in a neighborhood of each point. Li et al. used their model for segmentation and bias correction of magnetic resonance images (MRIs) with promising results.

According to this study, previous results and our case of study, we choose the level set method to segment breast mass.

\subsection{Level Set method}

The level set method is a generic numerical method for evolving fronts in an implicit form. The image segmentation problem can be formulated and solved with the level set method principally based on wellestablished mathematical theories, including calculus of variations and partial differential equations (PDE). Consider a curve/interface $\Gamma$ in movement in a region $\Omega$. $\Gamma$ is the zero level of a function $\varphi$ of higher dimension usually called a level set function. The interface in movement at point $\mathrm{x}$ at the instant $\mathrm{t}$ is defined as:

$$
\Gamma(\mathrm{t})=\{(x, t) \mid \varphi(\mathrm{x}, \mathrm{t})=0\}
$$

With $\varphi$ is positive inside $\Omega$, negative outside, and null on $\Gamma$. Items of this interface will move towards normal (gradient $\nabla \varphi$ ) with a speed $v$ according to the following equation:

$$
\frac{\partial \varphi}{\partial t}+v \cdot \nabla \varphi=0
$$

In fact, only the normal component of $\mathrm{v}$ account $v_{N}=v \cdot \frac{\nabla \varphi}{|\nabla \varphi|}$, With $|\nabla \varphi|$ is the euclidean norm, thus equation (1) becomes:

$$
\frac{\partial \varphi}{\partial t}+v_{N} \cdot|\nabla \varphi|=0
$$

The results of segmentation were validated by two radiologists. Two examples of both malignant and benign lesions are shown in the following figures.
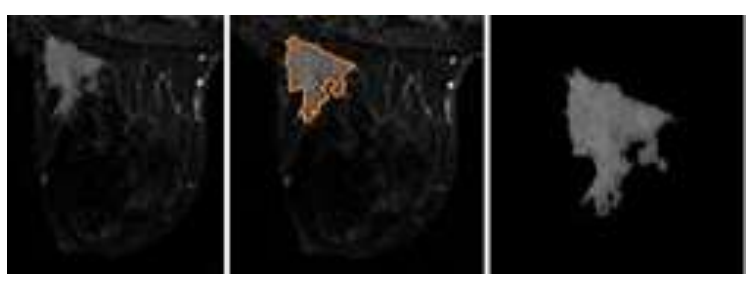

Figure 1: Original MRI, segmented malignant lesion and lesion extracted respectively from left to right.
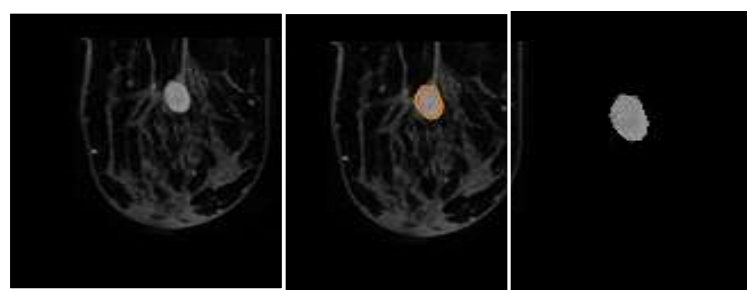

Figure 2: Original MRI, segmented benign lesion and lesion extracted respectively from left to right.

\subsection{Feature extraction}

Three kinds of characteristics could be extracted from the segmented tumors (from MRI and DECEDM): textures features, shape characteristics and kinetic features. In this paper, 20 GLCM features and 4 shape features are extracted and defined as follows. 


\subsubsection{Texture features}

Texture is the result of local variations in brightness within one small area of an image. A set of Gray Level Co-occurrence Matrix (GLCM) features, such as Haralick's features (Haralick, 1973) (energy, entropy, correlation, inverse difference moment, inertia, cluster shade, cluster prominence, etc.) and other texture characteristics (dissimilarity, maximum probability, autocorrelation, inverse difference normalized INN, inverse difference moment normalized IDN) (Clausi, 2002), (Soh, 1999) are extracted from the co-occurrence matrix. GLCM represents how different combinations of pixel brightness values occur in an image, it depicts the joint frequencies of all pairwise combinations of gray levels $i$ and $j$ separated by a distance along a direction $\theta$ (Karthikeyan, 2014). The GLCM is normalized by the sum of all its elements to calculate the cooccurrence relative frequency between gray level i and $j$. Each entry in GLCM matrix is considered to be the probability that a pixel with value $i$ will be found adjacent to a pixel of value $\mathrm{j}$ as defined below (Albregtsen, 2008):

$$
\left[\begin{array}{cc}
p(1,1) & p(1,2) \ldots p(1, N) \\
p(2,1) & p(2,2) \ldots p(2, N) \\
\ldots \\
p(N, 1) & p(N, 2) \ldots p(N, N)
\end{array}\right]
$$

\subsubsection{Shape features}

A round mass with smooth and well-defined contour tends to be benign, whereas an irregular or speculated mass with undefined border tends to be malignant. Various shape descriptors exist in the literature, and they are categorized into two groups: contour-based shape descriptors (Fourier descriptor, wavelet descriptors, shape signatures, etc.) and region-based shape descriptors (geometric moments, Zernike moments, etc.). Four categories of shape descriptors including compactness, signature, Zernike moments and GFD were used to diagnose the lesions.

\subsubsection{Compactness}

Compactness is defined as the ratio of the square of surface area to the total volume of the lesion. It is used to quantify the connectedness of the portions in a lesion. A highly none convex lesion (malignant lesion) will have a high compactness index, however benign lesions have a low compactness values (Nie, 2008). Mathematical expression of compactness is as follows:

$$
\text { compactness }=\frac{4 \pi \cdot \text { area }}{\text { perimeter }^{2}}
$$

\subsubsection{Shape Signature}

The shape signature is expressed by the distance of boundary points from the centroid $(\mathrm{xc}, \mathrm{yc})$ of the shape.

$$
\mathrm{r}(\mathrm{k})=\sqrt{\left(\mathrm{x}(\mathrm{k})-\mathrm{x}_{\mathrm{c}}\right)^{2}+\left(\mathrm{y}(\mathrm{k})-\mathrm{y}_{\mathrm{c}}\right)^{2}}
$$

Where $x(n)$ and $y(n)$ are the coordinates of the pixels that make the shape boundary, and $\mathrm{x}_{\mathrm{c}}=$ $\frac{1}{\mathrm{~N}} \sum_{\mathrm{k}} \mathrm{x}(\mathrm{k}), \mathrm{y}_{\mathrm{c}}=\frac{1}{\mathrm{~N}} \sum_{\mathrm{k}} \mathrm{y}(\mathrm{k})$.

The signature is normalized by the maximum distance between the centroid and the $\mathrm{k}$ boundary points to be invariant to rotation and scale, $r_{\text {norm }}(k)=\frac{r(k)}{\max _{i} r(i)}$.

\subsubsection{Zernike moments}

Zernike moments have been utilized as object descriptors in several pattern recognition problems (Hwang, 2006), in edge detection $(\mathrm{Li}, 2010)$ and image retrieval applications $(\mathrm{Li}, 2009)$ with significant results. The Zernike moments are a set of descriptors obtained using complex kernel functions based on Zernike polynomials orthogonal to each other which helps to represent the properties of an image with no redundancy or overlap of information between the moments (Tahmasbi, 2011). Zernike moments of order $n$ with repetition $m$ are defined as:

$$
Z_{n m}=\frac{n+1}{\pi} \int_{0}^{2 \pi} \int_{0}^{1} f(\rho, \theta) V_{n m}^{*}(\rho, \theta) \rho d \rho d \theta
$$

Where $f(x, y)$ represents the mass shape and $V^{*}$ is the complex conjugate of function $\mathrm{V}$. defined as:

$$
V_{n, m}(\rho, \theta)=R_{n m}(\rho) e^{j m \theta},|\rho| \leq 1, j=\sqrt{-1},
$$

where $\mathrm{R}_{\mathrm{nm}}(\rho)=\sum_{\mathrm{s}=0}^{(\mathrm{n}-|\mathrm{m}|) / 2} c(\mathrm{n}, \mathrm{m}, \mathrm{s}) \rho^{\mathrm{n}-2 \mathrm{~s}} \quad$ and $\mathrm{c}(\mathrm{n}, \mathrm{m}, \mathrm{s})=\frac{(n-s) !}{s !\left(\left(\frac{(n+|m|)}{2}\right)-s\right) !\left(\left(\frac{(n-|m|)}{2}\right)-s\right) !}, \mathrm{n}$ and $\mathrm{m}$ are respectively the order and the repetition.

To compute Zernike moments from a digital image, the integral should be replaced by summations and the coordinates of the image must be normalized into $[0,1]$ by a mapping transform. The discrete form of the Zernike moments of an image size $\mathrm{N} \times \mathrm{N}$ is expressed as follows:

$$
\begin{aligned}
& \mathrm{Z}_{\mathrm{nm}} \\
& =\frac{n+1}{\lambda_{N}} \sum_{x=0}^{N-1} \sum_{y=0}^{N-1} f(x, y) R_{n m}\left(\rho_{x y}\right) e^{-j m \theta_{x y}}
\end{aligned}
$$


Where $0 \leq \rho_{x y} \leq 1$, and $\lambda_{N}$ is a normalization factor.The transformed distance $\rho_{x y}$ and the phase $\theta_{x y}$ at the pixel of $(\mathrm{x}, \mathrm{y})$ are given by: $\rho_{\mathrm{xy}}=$ $\frac{\sqrt{(2 \mathrm{x}-\mathrm{N}+1)^{2}+(\mathrm{N}-1-2 \mathrm{y})^{2}}}{\mathrm{~N}}, \theta_{\mathrm{xy}}=\tan ^{-1}\left(\frac{\mathrm{N}-1-2 \mathrm{y}}{2 \mathrm{x}-\mathrm{N}-1}\right)$.

\subsubsection{Generic Fourier Descriptors}

The GFD is extracted from spectral domain by applying 2-D Fourier transform on polar raster sampled shape image. It has no redundant features and allows multi-resolution feature analysis in both radial and angular directions (Zhang, 2002).

The GFD are based on the Polar Fourier defined as:

$$
\mathrm{F}(\rho, \theta)=\sum_{\mathrm{x}} \sum_{\mathrm{y}} \mathrm{I}(\mathrm{x}, \mathrm{y}) \mathrm{e}^{\left[2 \mathrm{2j \pi}\left(\frac{\mathrm{r}(\mathrm{x}, \mathrm{y})}{\mathrm{R}} \rho+\mathrm{v}(\mathrm{x}, \mathrm{y}) \theta\right)\right]}
$$

Where $\mathrm{r}(\mathrm{x}, \mathrm{y})$ and $\mathrm{v}(\mathrm{x}, \mathrm{y})$ are respectively the radius and angle of the polar coordinates of the point $(\mathrm{x}, \mathrm{y}), \mathrm{I}$ is the intensity function and the parameters $\rho$ and $\theta$ are bounded: $0 \leq \rho<R$ and $0 \leq \theta<T$, with $R$ and $T$ respectively the radial and the angular resolutions. Finally, the GFD is written:

$$
\mathrm{GFD}(\mathrm{m}, \mathrm{n})=\left\{\frac{|\mathrm{PF}(0,0)|}{\mathrm{M}_{11}}, \frac{|\mathrm{PF}(0,1)|}{|\mathrm{PF}(0,0)|} \ldots \frac{|\mathrm{PF}(\mathrm{m}, \mathrm{n})|}{|\mathrm{PF}(0,0)|}\right\}
$$

Where $\mathrm{m}$ and $\mathrm{n}$ are the radial and angular frequencies and $M_{11}$ is the $1^{\text {st }}$ order moment.

\subsubsection{Kinetic features}

Kinetic features are extracted from the Time Intensity Curve (TIC) of the segmented lesion. As we have mentioned previously, time intensity curve allows to divide lesions into malignant and benign according to their time to peak, an initial rise of signal intensity within 90 seconds $>90 \%$ is defined as rapid enhancement, which is highly suggestive of malignancy. Four kinetic features could be extracted from the Characteristic Kinetic Curve CKC (Maximum enhancement, Time to peak, Uptake rate and Washout rate) (Chen, 2006).

\section{FEATURE SELECTION AND CLASSIFICATION}

\subsection{Context of the study}

Pattern recognition methods are generally built independently. Their combination may lead to positive correlations, because they aim at achieving the same goal and are based on the same learning data. Many classifier combination systems have been proposed and compared in the literature, and a full presentation of most of these can be found in a reference book by Duda et al. (Duda, 2012).

Over the years many aggregation operators have been introduced. For most of these operators, the relative importance of a criterion, associated here to a feature, is represented in the final decision by a weight assigned to it. By assigning a weight not only to each participant of the aggregation, but also to each subset of participants, the operators based on the Choquet integral are able to capture this synergy in the associated Fuzzy measure (or capacity). Choquet integral allows to consider effectively the interactions between decision rules while providing a model of robust decision in the presence of little training data that can be even very inconsistent (Grabisch, 1996).

\subsection{Choquet Integral}

Let us consider $m$ classes $\mathrm{C}_{1} \ldots \mathrm{C}_{\mathrm{m}}$ and $n$ decision rules (DRs) $X=\left\{D_{1} \ldots D n\right\}$. As in Wendling and al., (Wendling, 2008) a decision rule (i.e criterion) is assumed to be a feature with associated metric. To classify a new pattern $x$, we need firstly for each decision rule $j$ and each class $i$, to compute a confidence degree $\Phi_{j}^{i}$. Then we combine all these partial confidence degrees into a global confidence degree given by $\Phi\left(C_{i} \mid x\right)=\mathrm{H}\left(\Phi_{1}^{i}, \ldots, \Phi_{n}^{i}\right)$. Finally, $x$ is assigned to the class for which the confidence degree is the highest.

Calculating Choquet integral requires the definition of a capacity or a fuzzy measure. Let us denote $\mathrm{P}$ as the power set of $X$, i.e the set of all subsets of $X$. Capacity or a fuzzy measure on $\mathrm{X}$, is a function $\mu$ : $\mathrm{P}(\mathrm{X}) \rightarrow[0,1]$ which verifies $: \mu(\varnothing)=0, \mu(\mathrm{X})=1$ and $\mu(\mathrm{A}) \leq \mu(\mathrm{B})$ if $\mathrm{A} \subseteq \mathrm{B}$ (monotony).

The Choquet Integral of $\vec{\phi}=\left[\begin{array}{ll}\phi_{1} \ldots & \phi_{n}\end{array}\right]^{\mathrm{t}}$ noted $\mathrm{C}_{\mu}(\vec{\phi})$, is defined as :

$$
\mathrm{C}_{\mu}(\vec{\phi})=\sum_{\mathrm{j}=1}^{\mathrm{n}} \phi_{(\mathrm{j})}\left[\mu\left(\mathrm{A}_{(\mathrm{j})}\right)-\mu\left(\mathrm{A}_{(\mathrm{j}+1)}\right)\right]
$$

Where $A_{(j)}=\{(j), \ldots(n)\}$ represents $[\mathrm{j} \ldots \mathrm{n}]$ associated criteria in increasing order and $\mathrm{A}(\mathrm{n}+1)=\emptyset$.

For a thorough description of the Choquet integral, the reader may refer to (Grabisch, 1996).

\subsection{Learning Step}

The calculation of the Choquet integral requires the assessment of any set of $\mathrm{P}(\mathrm{X})$. The main problem is giving a value to the sets that have more than three elements while keeping the monotonic property of the 
integral. Generally the problem is translated to another minimization problem, which is usually solved using the Lemke method. M. Grabisch (Grabisch, 1996) has shown that such an approach may be inconsistent when using a small number of samples and has proposed an optimal approach based on a gradient algorithm. In the approach it is assumed that in the absence of any information, the most reasonable way of aggregation is the arithmetic mean. This algorithm tries to minimize the mean square error between the values of the Choquet integral and the expected values.

\subsection{Extraction step}

Once the fuzzy measure is learned, it is possible to interpret the contribution of each decision criterion in the final decision. We adapt here the strategy proposed in (Mazaud, 2007) to our breast cancer diagnosis problem. Several indices can be extracted from the fuzzy measure, helping to analyze the behavior of decision criteria. The importance of each criterion is based on the definition proposed by Shapley in the game theory (Shapley, 1953). Let us consider a fuzzy measure $\mu$ and a decision criterion $\mathrm{D}_{\mathrm{i}}$ :

$$
\begin{gathered}
\sigma\left(\mu, D_{i}\right)=\frac{1}{n} \sum_{t=0}^{n-1} \frac{1}{\left(\begin{array}{c}
n-1 \\
t
\end{array}\right)} \sum_{T \subseteq N \backslash D_{i}}\left[\mu\left(T \cup D_{i}\right)\right. \\
-\mu(T)]
\end{gathered}
$$

A decision criterion with an importance index value less that $1 / \mathrm{n}$ can be interpreted as having a low impact in the final decision. Otherwise an importance index greater than $1 / \mathrm{n}$ describes an attribute more important than the average. The interaction index, also called the Murofushi and Soneda index (Murofushi, 1991), (Murofushi, 1993) represents the positive or negative degree of interaction between two decision criteria. If the fuzzy measure is nonadditive, then some criteria interact. The marginal interaction between $D_{i}$ and $D_{j}$, conditioned to the presence of elements of combination $\mathrm{T} \subseteq \mathrm{X} \backslash \mathrm{D}_{\mathrm{i}} \mathrm{D}_{\mathrm{j}}$ is given by:

$$
\begin{aligned}
& \left(\Delta_{D_{i} D_{j}} \mu\right)(T)=\mu\left(T \cup D_{i} D_{j}\right)+\mu(T)- \\
& \mu\left(T-D_{i}\right)-\mu\left(T-D_{j}\right)
\end{aligned}
$$

After averaging it over all the subsets of $T \subseteq$ $X \backslash D_{i} D_{j}$ the assessment of the interaction index of $D_{i}$ and $D_{j}$, is defined by:

$$
\begin{aligned}
& I\left(\mu, D_{i} D_{j}\right) \\
& =\sum_{T \subseteq X \backslash D_{i} D_{j}} \frac{(n-t-2) ! t !}{(n-1) !}\left(\Delta_{D_{i} D_{j}} \mu\right)(T)
\end{aligned}
$$

We propose to sort the decision criteria with increasing order, by considering the values they reach, using a linear combination of importance and interaction indexes $f_{D_{i}}$.

Let us first consider the global impact of interactions of order 2, or the normalization factor K, as follows:

$$
K=2 \times \sum_{k=1, n} \sum_{j=k+1, n}\left|I\left(\mu, D_{k} D_{j}\right)\right|
$$

If $\mathrm{K}$ is close to $0(\mathrm{~K} \approx 0)$, then no (or few) interactions exist between the decision criteria, as they are independent. The Choquet integral relies on a weighted sum and the impact of each decision criterion can be directly assessed by taking its Shapley values: $f_{D_{i}=} \sigma\left(\mu, D_{i}\right)$.

Otherwise, the assessment is performed using the importance and interaction indices. It is calculated by integrating the interaction impact of $D_{i}$ relatively to the minimum of global interaction reached by one decision criterion, noted $\mathrm{M}$, as follows:

$$
\begin{gathered}
f_{D_{i}}=n \times \sigma\left(\mu D_{i}\right) \times\left(\sum_{j=1, n} I\left(\mu D_{i} D_{j}\right)-\right. \\
M) / K \\
M=\min \left\{\sum_{j=1, n}\left|I\left(\mu D_{i} D_{j}\right)\right|\right\}_{k=1, n}
\end{gathered}
$$

The decision criterion having the least influence on the final decision, and interacting the least with the other criteria is assumed to blur the final decision.

\section{EXPERIMENTAL RESULTS AND DISCUSSION}

\subsection{Database}

A breast MRI database, whose examinations date from 2014 and 2015, is used in this study. The images are provided by the hospital Georges Pompidou radiology department. An expert radiologist identified the corresponding physical lesions based on visual criterion and biopsy-proven reports. The MRI database consists of 58 mass lesion in 40 patients of which 14 lesions are benign and 44 lesions are malignant. Know that, by virtue of having MRI performed as part of the clinical exam, these lesions could be assumed to be difficult to interpret. The 
segmentation of breast images was validated by two experts.

\subsection{Results}

It is important to note that only subset of features among the set of features presented before are relevant despite they were usually used in the literature. Most of them are based on same mathematical process and are redundant and blurred the results. The aim of the approach is to automatically extract a subset of pertinent features adapted to the studied application. Each feature is assumed to be a decision rule. That is descriptor and associated metric to be consistent with the proposed methodology. Leave-one-out is performed as few data are processed and to provided non-biased results. For a lack of clarity we will study the subset of nine remaining features providing a score of $100 \%$ after selecting step (that is Energy, Entropy, Homogeneity, Correlation, Contrast, Cluster Prominence, Cluster Shade, Compactness, and Zernike moments). The analyze of Choquet lattice allows to attribute to each feature a score based on its importance index (Shapley index) and its global interaction impact. At each epoch the weakest feature is removed and the new rate of correct classification is calculated.

The following table shows the variation of correct classification rates (CCR) by running several methods.

Table 1: Correct classification rates (CCR) obtained on subsets of features using well-known classifiers.

\begin{tabular}{|c|c|c|c|c|c|c|}
\hline $\begin{array}{l}\text { Methods } \\
\text { /Remove } \\
\text { d feature }\end{array}$ & $\mathrm{F}$ & Kmeans & Knn & ANN & SVM & $\begin{array}{l}\text { Cho } \\
\text { quet }\end{array}$ \\
\hline & 9 & 74 & 90 & 76 & 87 & 100 \\
\hline Energy & 8 & 81 & 90 & 76 & 84 & 95 \\
\hline Entropy & 7 & 74 & 90 & 81 & 84 & 96 \\
\hline ClusterP & 6 & 29 & 90 & 59 & 79 & 94 \\
\hline ClusterS & 5 & 26 & 86 & 74 & 79 & 92 \\
\hline Homog & 4 & 74 & 84 & 72 & 81 & 92 \\
\hline Contrast & 3 & 79 & 92 & 71 & 79 & 93 \\
\hline Zernike & 2 & 21 & 92 & 76 & 79 & 89 \\
\hline
\end{tabular}

$\mathrm{F}=$ number of used features. Kmeans $=\mathrm{K}$-means clustering. $\mathrm{Knn}=\mathrm{k}$-nearest neighbor. ANN = artificial neural network. $\mathrm{SVM}=$ support vector machine. Choquet $=$ Choquet integral. ClusterP $=$ cluster prominence. ClusterS $=$ cluster shade. Homog = homogeinity.

Kmeans and SVM classifiers have a better correct classification rate CCR ( $81 \%$ and $84 \%$ respectively) with 8 features by removing energy, Knn gives a better recognition rate (92\%) with only 3 features kept (Zernike, compactness and correlation). Knn, SVM and Choquet integral provide a better CCR ( $81 \%, 84$ and $96 \%$ respectively) with two removed features, energy and entropy, but Choquet integral out performs all the classification methods with $96 \%$ of CCR. These results attest the good behavior of our method based on Choquet integral. Furthermore the importance of the feature can be assessed at each level (Table 2) as well as their interaction (Table 3).

Table 2: An example of the assessment of the importance of decision rules (Shapley) associated to a set of 7 features (table 1).

\begin{tabular}{|l|l|l|l|l|l|l|l|}
\hline Shapley & F1 & F2 & F3 & F4 & F5 & F6 & F7 \\
\hline values & 0.76 & $\mathbf{1 . 0 1}$ & $\mathbf{1 . 0 9}$ & 0.78 & 0.74 & $\mathbf{1 . 4 6}$ & $\mathbf{1 . 1 5}$ \\
\hline
\end{tabular}

Table 3: An example of the assessment of the positive and negative interactions between decision rules (second order) associated to a set of 7 features (table 1). Obviously table is symmetric.

\begin{tabular}{|c|c|c|c|c|c|c|c|}
\hline Int. 2 & F1 & F2 & F3 & F4 & F5 & F6 & F7 \\
\hline F1 & --- & -0.18 & -0.11 & 0.08 & 0.31 & 0.26 & 0.64 \\
\hline F2 & -0.18 & --- & -0.16 & 0.41 & 0.01 & 1.09 & -0.34 \\
\hline F3 & -0.11 & -0.16 & --- & 0.00 & 0.42 & -0.33 & 0.18 \\
\hline F4 & 0.08 & 0.41 & 0.00 & --- & 0.54 & -0.12 & 0.74 \\
\hline F5 & 0.31 & 0.01 & 0.42 & 0.54 & --- & 0,02 & 0.70 \\
\hline F6 & 0.26 & 1.09 & -0.33 & -0.12 & 0.02 & --- & -0.93 \\
\hline F7 & 0.64 & -0.34 & 0.18 & 0.74 & 0.70 & -0.93 & -- \\
\hline
\end{tabular}

\section{CONCLUSIONS}

In this study, we combined selected textural and morphological features to improve the overall performance of breast MRI in a computer-aided system. We opted for Choquet integral, which provides a robust model of decision in the presence of little training data, to select the most pertinent features. The results are very encouraging $(\mathrm{CCR}=$ $96 \%$ ), despite using a small data set. Further works are devoted to the extension of this model to process with other subsets of features extracted from breast MRI (kinetic features, risk factor, etc.) and from DECEDM and to merge a set of features in order to reach a better tumor recognition and classification which lead to a better decision. Such a generic system 
can be useful for other type of dataset for cancer recognition.

\section{REFERENCES}

Albregtsen, F., 2008. Statistical Texture Measures Computed from Gray Level Co-occurrence Matrices. Image Processing Laboratory Department of Informatics University of Oslo.

Chen, W., Giger, ML., Bick, U., 2006. A Fuzzy C-Means (FCM)-Based Approach for Computerized Segmentation of Breast Lesions in Dynamic ContrastEnhanced MR Images. Academic Radiology. Volume 13 , issue 1 , pages $63-72$.

Chen, W., Giger, ML., Bick, U., 2006. Automatic identification and classification of characteristic kinetic curves of breast lesions on DCE-MRI. Med Phys. 33(8), pages 2878-87.

Chen, W., Giger, ML., Lan, L., et al., 2004. Computerized interpretation of breast MRI: investigation of enhancement-variance dynamics. Med Phys. Volume 31, pages 1076-1082.

Clausi, DA., 2002. An analysis of co-occurrence texture statistics as a function of grey level quantization. Can. J. Remote Sensing. Volume 28, issue 1, pages 45-62.

Duda, RO., Hart, PE., Stork, DG., 2012. Pattern classification. John Wiley \& Sons.

Grabisch, M., 1996. The application of fuzzy integrals in multicriteria decision making. Eur. J. Oper. Res. Volume 89, issue 3, pages 445-456.

Haralick, RM., Shanmuga, K., Dinstein, I., 1973. Textural features for image classification. IEEE Trans Syst Man Cybern; Smc3 (6):610-21.

Hwang, S.K., Kim, W.Y., 2006. A novel approach to the fast computation of Zernike moments. Pattern Recognition. Volume 39 pages 2065-2076.

Karthikeyan, S., Rengarajan, N., 2014. Performance analysis of gray level co-occurrence matrix texture features for glaucoma diagnosis. American Journal of Applied Sciences. Volume 11, pages 248-257.

Li, C., Kao, CY., Gore, JC., Ding, Z., 2008. Minimization of region-scalable fitting energy for image segmentation. IEEE Transactions on Image Processing. Volume 17, issue 10, pages 1940-1949.

Li, C., Huang, R., Ding, Z., Gatenby, JC., Metaxas, DN., Gore, JC., 2011. A level set method for image segmentation in the presence of intensity inhomogeneities with application to MRI. IEEE Transactions on image processing. Volume 20, issue 7.

Li, Sh., Lee, MCh, Pun, Ch.M., 2009. Complex Zernike moments features for shape based image retrieval. IEEE Transactions on Systems, Man and Cybernetics, Part A: Systems and Humans 1. Volume 39, pages 227237.

Li, X., Li, L., Lu, H., Chen, D., Liang, Z., 2003. Inhomogeneity Correction for Magnetic Resonance Images with Fuzzy C-Mean Algorithm. Proc. SPIE Int. Soc. Opt. Eng., Volume 5032, pages 995-1005.
Li, X., Song, A., 2010. A new edge detection method using Gaussian-Zernike moment operator. Proceedings of the IEEE, 2nd International Asia Conference on Informatics in Control, Automation and Robotics, pages 276-279.

Liney, GP., Sreenvias, M., Garcia-Alvarez, R., et al., 2006. Breast lesion analysis of shape technique: semiautomated vs. manual morphological description. J Magnetic Resonance Imaging. Volume 23, pages 493498.

Mazaud C., Rendek J., Bombardier V., Wendling L., "A feature selection method based on Choquet Integral and Typicality Analysis", IEEE International Conference on Fuzzy Systems, London (UK), 6p, 2007.

Murofushi, T., Sugeno, M., 1991. A theory of fuzzy measures: Representations, the Choquet integral, and null sets. J. Math. Anal. Appl. Volume 159, issue 2, pages 532-549.

Murofushi, T., Soneda, S., 1993. Techniques for reading fuzzy measures (iii): Interaction index. Proc. 9th Fuzzy Syst. Symp., Sapporo, Japan. Pages 693-696.

Nie, K., 2008. Quantitative Analysis of Lesion Morphology and Texture Features for Diagnostic Prediction in Breast MRI. Academic Radiology, volume 15, issue 12, pages 1513-1525.

Shapley, L., 1953. A value for n-person games. In Contributions to the Theory of Games, Annals of Mathematics Studies, H. Khun and A. Tucker, Eds. Princeton, NJ: Princeton Univ. Press, pages 307-317.

Soh, LK., Tsatsoulis, C., 1999. Texture analysis of SAR sea ice imagery using gray level co-occurrence matrices. IEEE Transactions on Geoscience and Remote Sensing. Volume 37, issue 2, pages 780-795.

Tahmasbi, A., 2011. Classification of benign and malignant masses based on Zernike moments. Computers in Biology and Medicine. Volume 41, pages 726-735.

Wang, TC., Huang, YH., Huang, CS., Chen, JH., Huang, GY., Chang, YC., Chang, RF., 2014. Computer-aided diagnosis of breast DCE-MRI using pharmacokinetic model and 3-D morphology analysis. Magnetic Resonance Imaging. Volume 32, pages 197-205.

Wendling L., Rendek J., Matsakis P., 2008. Selection of suitable set of decision rules using Choquet integral, Statistic Pattern Recognition, S+SSPR'08, pp10.

Winzenrieth, R., Claude, I., Hobatho, MC., Pouletaut, P., Sebag, G., 2003. Comparaison de deux méthodes de segmentation par contours actifs : les snakes et les level sets pour la segmentation d'IRM de hanche.

Zhang, D., Lu, G., 2002. Generic Fourier descriptor for shape-based image retrieval. Multimedia and Expo, ICME '02. Proceedings. IEEE International Conference. Volume1, pages 425-428.

Zhang, DQ., Chen, S., Pan, ZS., Tan, KR., 2003. Kernel based fuzzy clustering incorporating spatial constraints for image segmentation. IEEE International conference on Machine Learning and Cybernetics. Volume 4, pages 2189-2192. 